\title{
Morphology of the dural venous complex of skull base in human ontogenesis
}

\author{
Maryna Kornieieva* \\ Anatomy, Histology, and Embryology Department, AUC School of Medicine, Lowlands, Sint Maarten, Netherlands Antilles
}

\begin{abstract}
The development of the dural venous complex of the skull base formed by the cavernous, intercavernous, and petrous dural sinuses and their connections with the intra- and extracranial veins and venous plexuses, was investigated on 112 premature stillborn human fetuses from 16 to 36 weeks of gestation by methods of vascular corrosion casting.

It was established that the main intracranial dural canals approach similar to the mature arrangement at the very beginning of the early fetal period. In fetuses 16 weeks of gestation, the parasellar dural venous complex appeared as a plexiform venous ring draining the venous plexus of the orbits into the petrous sinuses. The average diameter of dural canals progressively enlarged and reached its maximum value $2.2 \pm 0.53 \mathrm{~mm}$ approaching the 24th week of gestation. This developmental stage is characterized by the intensive formation of the emissary veins connecting the cavernous sinus with the extracranial venous plexuses. Due to the particular fusion of the intraluminal canals, the average diameter of the lumen gradually declined to reach $1.9 \pm 0.54$ mm in 36 -week-old fetuses. By the end of the fetal development, $21.3 \%$ of fetuses featured a considerable reduction of the primary venous system with the formation of the one-canal shaped dural venous sinuses, obliteration of several tributaries, and decreased density of the extracranial venous plexuses. In the other third of fetuses, the enhanced venous basal complex with the multi-canal shape of dural sinuses, and abundance of tributaries and anastomoses persisted thought the whole antenatal period.
\end{abstract}

Thus, the genetically determined pace of reduction of the primary venous plexuses determines the final shape of the dural venous system and its compensatory abilities.

\section{Introduction}

At the beginning of the early fetal period, the dural venous complex of the skull base is represented by two cavernous sinuses connected via the anterior and posterior intercavernous sinuses, the superior and inferior petrosal sinuses, and the marginal sinus located around the foramen magnum. During the early fetal period, the basilar dural complex dominates over the sinuses of calvaria draining the intensively developing structures of the orbit. The progressive enlargement of the cerebral hemispheres on the following stages of fetal development triggers significant reconstruction of venous outflow canals with the establishment of numerous intra- and extracranial anastomoses $[1,2]$. Although the early embryogenesis of the cranial venous system is relatively well described in the previously mentioned works, the clinically relevant reorganization of the dural sinuses of the skull base at the early and late fetal trimesters have not got proper attention yet.

The reconstruction and maturation of the fetal dural system is an important concept critical for analysis and successful treatment of benign dural malformations in neonates, referred to as the dural arteriovenous shunts. The dural arteriovenous shunts in neonates are rare but potentially fatal entities [3]. The symptoms of the dural arteriovenous shunts largely depend on the type of variability of the basilar dural complex, the drainage patterns of the shunt, and the coexistence with venous thrombosis [3-6]. Analysis of 30 clinical cases with this pathology in neonates and infants shows that only half of them had received transvascular embolization treatment, preferably via the arterial tree. All untreated babies died, whereas $75 \%$ of treated ones had good neurological outcomes [3]. Comparison of outcomes for 27 adult patients treated by transvenous and transarterial embolization methods allowed the author to advocate the first approach, as it gave $87 \%$ of positive results [7]. Presumably, the intravenous approach would improve the outcomes in infants, too. Detailed morphologic analysis of the development and maturation of the fetal dural venous system could open up new possibilities for the treatment of such neonates.

Present morphologic research is designed to evaluate the developmental reconstructions of the dural venous system of the skull base in human fetuses from 16 to 36 weeks of gestation and to describe the range of variability of the cavernous, intercavernous and petrous dural sinuses established in preborn fetuses.

\section{Materials and methods}

This study was carried out on 112 premature stillborn human fetuses (64 males and 48 females) aged between 16-36 weeks of gestation. The gestational age was estimated by the last menstrual period of the mother and by measuring of weight and length of the fetus. All specimens with congenital malformations of the head and central nervous system were excluded from the study. The research had been conducted at the

${ }^{*}$ Correspondence to: Maryna Kornieieva, American University of Caribbean School of Medicine, Lowlands, Sint Maarten, Netherlands Antilles, Tel: 721-5452298 Ext: 4042355, Cell: +1(721)5591342; E-mail: mkornieieva@aucmed.edu

Key words: cavernous sinus, intercavernous sinuses, superior petrous sinus, inferior petrous sinus, human fetal development

Received: September 09, 2019; Accepted: September 16, 2019; Published: September 19, 2019 
Lugansk State Medical University (Ukraine) following International Norms of Biological Research and was approved by the Institutional Ethics Committee (Protocol number 10.978.03 from 23.01.2007).

In the selected specimens, the extended thoraco- and pericardiotomy were performed, exposing the surface of the heart. A fine catheter introduced into the superior vena cava via the right atrium of the heart was used to inject 1:3 mixture of acrylate monomer with dichloromethane into the venous system until complete filling of the superficial veins of the scalp. The oil-based blue paint was added to the plastic to color the vessels. After the injection, a fetus was immersed into $10 \%$ formaldehyde for 5-7 days. Then, the head of the specimen was placed into the concentrated hydrochloric acid for 10-14 days for corrosion of the soft tissues. An obtained plastic cast of the venous system of the head was carefully rinsed under running water.

The components of the dural venous complex of the skull base were studied through a monocular biological microscope with tenfold magnification (Levenhuk 5S NG). The diameter and length of the cavernous, anterior and posterior intercavernous, and superior and inferior petrous sinuses were measured under a microscope eyepiece micrometer with calibration measuring scale ruler reticle $0.1 \mathrm{~mm}$. The intracranial tributaries and emissary veins attached to the sinuses were enumerated. The mean and standard deviation of the resultant data were calculated for the early (16-20-24 weeks) and late (28-32-36 weeks) trimesters of the fetal development separately. The difference of results was evaluated with paired t-test (2-tailed). A p-value 0.05 and less was considered as statistically significant.

\section{Results}

It was established that the main intracranial dural canals approximate the mature arrangement at the very beginning of the early fetal period (Figure 1). In fetuses 16 weeks of gestation, the parasellar dural venous complex appeared as a plexiform venous ring formed by paired cavernous sinuses connected via anterior and posterior intercavernous sinuses.

The lumen of the cavernous sinus appeared as a collection of tiny venous channels. The average diameter of the sinus underwent irregular changes during the early fetal period. It slightly decreased by the fifth month of gestation up to $1.5 \pm 0.69 \mathrm{~mm}$, but progressively enlarged and reached its maximum value $(2.2 \pm 0.53 \mathrm{~mm})$ approaching the 24th week of gestation (Table 1). In the late fetal period, the intraluminal venous channels became much larger through expansion and unification, resulting in a single-canal shape in $36 \%$ of fetuses. However, in the majority of specimens, the plexiform arrangement of the sinus persisted through the whole antenatal development (Figure 2 ). Due to the particular fusion of the canals, the average diameter of the lumen gradually declined, reaching $1.9 \pm 0.54 \mathrm{~mm}$ in 36-week-old fetuses. The length of the cavernous sinus steadily increased with the fetus growth; the periods of high intensity felt on the sixth and eight months.

During the early fetal period, the cavernous sinus primarily drained the orbital venous plexus, while the connections with the deep cerebral veins had been establishing through the sixth and seventh months of gestation. The late fetal period was characterized by the intensive formation of the emissary veins connecting the cavernous sinus with the extracranial venous plexuses. The inferior and superior petrous sinuses provided the main outflow from the cavernous sinus to the transverse and sigmoid sinuses during the whole fetal period.

The anterior and posterior intercavernous sinuses connected the lateral cavernous compartments in front and behind the sella turcica

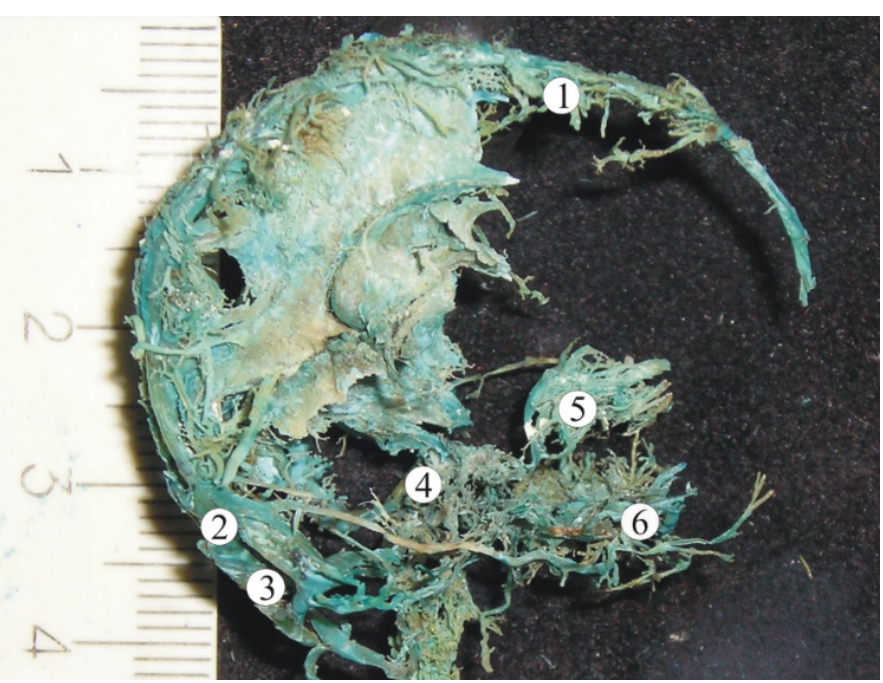

Figure 1. A plastic cast of the venous system of the head of a human fetus $16^{\text {th }}$ week of gestation revealing well-formed superior sagittal (1), transverse (2), and sigmoid (3) sinuses. The cavernous venous complex of the skull base (4) has a plexiform shape. The veins of the orbit (5) and veins of the face region (6) are all drained by the sinuses of skull base at this stage of gestation

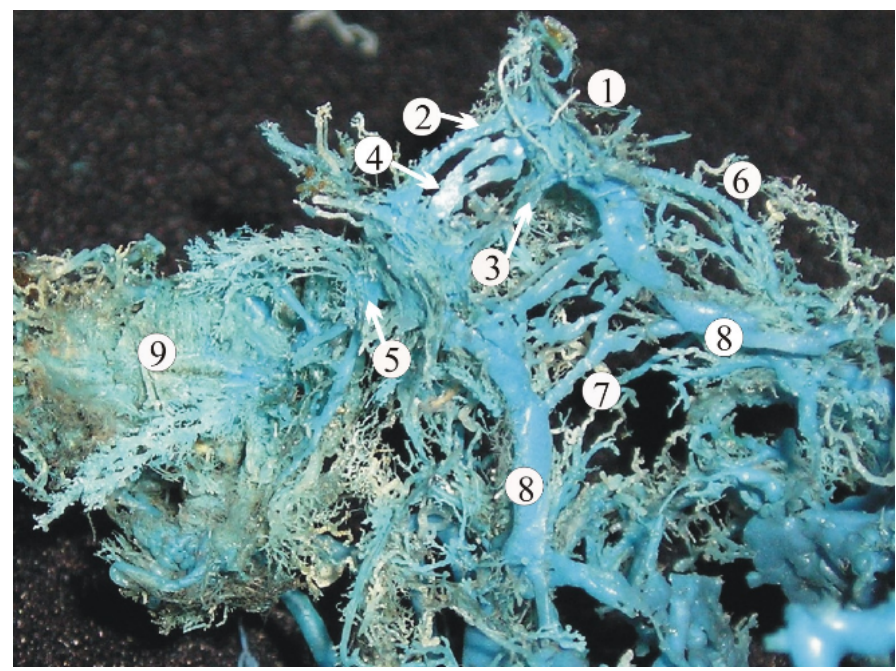

Figure 2. A plastic cast of the venous dural complex of the skull base of a 24-week human fetus showing the cavernous sinus $(1)$, two-canal shaped anterior $(2,4)$ and posterior $(3)$ intercavernous sinuses. The superior (6) and inferior petrous sinuses (8) continues with the caudal part of the lateral cavernous sinus. The intra-dural venous vessels of the basilar venous plexus (7) connect the inferior petrous sinuses running across the clivus of the occipital bone. The emissaries of the foraminae rotundum and ovale (5) connect the cavernous sinus with the well-developed pterygoid venous plexus (9)

from the very beginning of the fetal development. The dynamic of their formation reflects the pattern described above to some extent. While the length of the sinuses gradually increased through the early and late fetal trimesters, the diameter attained its pick by 28 - 32 weeks of gestation with following stable reduction (Table 1). The anterior intercavernous sinus had a double-canal shape in the majority of 16 -24-week fetuses with the diameter from 0.2 to $0.8 \mathrm{~mm}$ (Figure 2). In $30 \%$ of cases, the upper canal received tributaries from the well-developed orbital plexus. The lower channel featured the presence of 2 to 4 emissary veins draining the submucous venous plexus of the sphenoidal sinus (Figure 3). As the development progressed to the late fetal period, one of the channels became plexiform, gradually losing connection with the main cavernous trunks, and underwent complete reduction. In the majority of fetuses, the posterior intercavernous sinus had a single-canal shape 
Table 1. Dynamics of the development of the cavernous dural complex in human ontogenesis ${ }^{\mathrm{a}}$

\begin{tabular}{|c|c|c|c|c|c|c|}
\hline \multirow{2}{*}{$\begin{array}{l}\text { Stage of gestation, } \\
\text { weeks }\end{array}$} & \multicolumn{2}{|c|}{ ACS } & \multicolumn{2}{|c|}{ LCS } & \multicolumn{2}{|c|}{ PCS } \\
\hline & Diameter & Length & Diameter ${ }^{b}$ & Length $^{b}$ & Diameter & Length \\
\hline \multirow[t]{2}{*}{16} & $0.1-0.3$ & $3.2-3.4$ & $1.8-1.9$ & $3.1-4.3$ & $0.1-0.3$ & $3.1-3.6$ \\
\hline & $0.2 \pm 0.08^{* *}$ & $3.3 \pm 0.08 \mathrm{NS}$ & $1.8 \pm 0.04 *$ & $3.9 \pm 0.54^{*}$ & $0.2 \pm 0.08^{*}$ & $3.4 \pm 0.07^{* *}$ \\
\hline \multirow[t]{2}{*}{20} & $0.4-0.8$ & $3.7-5.6$ & $0.5-2.4$ & $3.7-5.9$ & $0.2-0.6$ & $3.4-5.9$ \\
\hline & $0.6 \pm 0.13^{* *}$ & $4.8 \pm 0.83 *$ & $1.5 \pm 0.69^{*}$ & $4.6 \pm 0.80^{* *}$ & $0.4 \pm 0.15^{* *}$ & $4.5 \pm 0.89^{* *}$ \\
\hline \multirow[t]{2}{*}{24} & $0.3-0.9$ & $4.0-6.8$ & $1.4-3.2$ & $4.1-6.8$ & $0.2-1.4$ & $4.1-6.8$ \\
\hline & $0.5 \pm 0.13 * * *$ & $5.4 \pm 0.30^{*}$ & $2.2 \pm 0.53 *$ & $5.5 \pm 0.93 *$ & $0.5 \pm 0.355^{*}$ & $5.4 \pm 0.74 \mathrm{NS}$ \\
\hline \multirow[t]{2}{*}{28} & $0.4-1.8$ & $6.6-7.8$ & $1.2-2.9$ & $4.2-6.9$ & $0.3-0.8$ & $6.1-7.9$ \\
\hline & $0.8 \pm 0.57^{*}$ & $6.9 \pm 0.28 * *$ & $2.1 \pm 0.61 *$ & $5.9 \pm 1.08 \mathrm{NS}$ & $0.5 \pm 0.15^{*}$ & $6.86 \pm 0.61^{*}$ \\
\hline \multirow[t]{2}{*}{32} & $0.6-1.3$ & $5.4-9.7$ & $1.3-2.3$ & $5.5-8.4$ & $0.4-1.2$ & $5.3-9.3$ \\
\hline & $0.8 \pm 0.27 *$ & $7.1 \pm 1.91 * *$ & $2.02 \pm 0.4 * *$ & $6.2 \pm 1.23 * *$ & $0.6 \pm 0.29 * *$ & $7.02 \pm 1.66^{* * *}$ \\
\hline \multirow[t]{2}{*}{36} & $0.4-1.0$ & $6.4-9.6$ & $0.7-2.7$ & $4.7-9.3$ & $0.4-0.8$ & $7.0-8.6$ \\
\hline & $0.6 \pm 0.25^{* *}$ & $7.9 \pm 1.11 * *$ & $1.8 \pm 0.71 * * *$ & $7.03 \pm 1.3^{* *}$ & $0.5 \pm 0.16^{* * *}$ & $8.1 \pm 0.64 * *$ \\
\hline
\end{tabular}

Abbreviations: ACS, anterior cavernous sinus; LCS, lateral cavernous sinus; PCS, posterior cavernous sinus.

${ }^{a}$ Mean $(\mu) \pm \operatorname{SD}(\sigma \eta-1), \mathrm{mm}$

${ }^{b}$ Mean value for the right and left lateral cavernous sinuses

Significance levels: ${ }^{* * *} \mathrm{p}<0.001 ; * * \mathrm{p}<0.01 ; * \mathrm{p}<0.5$; NS, no statistical significance

with the diameter up to $0.2-0.4 \mathrm{~mm}$ that slightly increased by the 24 th week of gestation. At the same stage, the anastomotic connections of the posterior intercavernous sinus with the basilar venous plexus over the clivus of occipital bone were traced.

In fetuses 16 weeks of gestation, the superior petrous sinus was represented by a single canal $0.6-0.9 \mathrm{~mm}$ in diameter (Table 2). Along its length, it received 2-3 cerebral veins and ran posterolaterally towards the convexity of the sigmoid sinus. The length of the superior petrous sinus gradually increased from $10.2 \mathrm{~mm}$ at the beginning of the early fetal period to $23.6 \mathrm{~mm}$ approaching the 36 th week of development. In fetuses 20 - 24 weeks of gestation, a dense dural venous network developed in the upper wall of the dural sinus which continued medially with the venous plexus of the tentorium cerebellum (Figure 4). Only at this gestational stage, a particular or full division of the lumen of the superior petrous sinus into two or three canals was registered in $27 \%$ of fetuses. All preborn fetuses featured the single-canal shape of the superior petrous sinus.

Close relationships of the superior petrous sinus with the superficial middle cerebral vein were observed in the study. The vein usually forms in the depth of the lateral sulcus receiving tributaries from the cortical veins of the anterior temporal and lower frontal lobes and typically drains into the cavernous sinus. In $27 \%$ of our specimens, the superficial middle cerebral vein anastomosed with the superior ophthalmic vein then ran posteriorly crossing the superior petrous sinus at the point between its anterior and middle thirds and opened into the transverse or sigmoid sinus (Figure 5). Persistence of such anatomic variability in adults occurs in $9 \%$ of cases and is classified as "underdeveloped type" according to Suzuki, et al. [8].

The inferior petrous sinus connected the cavernous sinus with the sigmoid one, or with the upper bulb of the jugular vein. The inferior petrosal sinus had a single-canal shape predominantly. Its length increased through the antenatal period from $7.2 \mathrm{~mm}$ to $16.7 \mathrm{~mm}$, and diameter expanded from $0.9 \mathrm{~mm}$ in 16 -week fetuses up to $1.4 \mathrm{~mm}$ in 36 -week ones. In $27 \%$ of the 20 -week fetuses, the inferior petrous sinus left the cranial cavity passing along with the internal jugular vein to the neck and entered the vein $1.2-2.5 \mathrm{~cm}$ below the skull base (Figure 6). Such variation often was balanced by the presence of anastomotic vein(s) connecting the internal jugular vein with the inferior petrous sinus within the jugular foramen. In such cases, the extracranial part of the sinus was considered as an inferior petrous vein.
Table 2. Dynamics of the development of the superior and inferior petrous sinuses in human ontogenesis ${ }^{\mathrm{a}}$

\begin{tabular}{|c|c|c|c|c|}
\hline \multirow{2}{*}{$\begin{array}{c}\text { Stage of } \\
\text { gestation, } \\
\text { weeks }\end{array}$} & \multicolumn{2}{|c|}{ SPS } & \multicolumn{2}{c|}{ IPS } \\
\cline { 2 - 5 } & Diameter & Length & Diameter & Length \\
\hline \multirow{2}{*}{$\mathbf{1 6}$} & $0.6-0.9$ & $10.2-10.7$ & $0.5-0.9$ & $7.2-9.8$ \\
\cline { 2 - 5 } & $0.71 \pm 0.13^{*}$ & $10.4 \pm 0.21^{*}$ & $0.63 \pm 0.2 \mathrm{NS}$ & $8.73 \pm 1.21^{* *}$ \\
\hline \multirow{2}{*}{$\mathbf{2 0}$} & $0.9-1.4$ & $16.0-19.1$ & $0.7-1.1$ & $10.4-14.0$ \\
\cline { 2 - 5 } & $1.18 \pm 0.15^{* *}$ & $16.6 \pm 1.4^{* * *}$ & $0.9 \pm 0.13^{*}$ & $12.9 \pm 2.12^{* *}$ \\
\hline \multirow{2}{*}{$\mathbf{2 4}$} & $1.1-1.7$ & $15.2-19.7$ & $0.9-1.4$ & $11.4-15.7$ \\
\cline { 2 - 5 } & $1.51 \pm 0.27 \mathrm{NS}$ & $18.03 \pm 2.38^{* *}$ & $1.08 \pm 0.25^{*}$ & $13.2 \pm 4.6^{*}$ \\
\hline \multirow{2}{*}{$\mathbf{2 8}$} & $1.2-2.2$ & $16.2-23.6$ & $0.8-1.2$ & $13.4-16.7$ \\
\cline { 2 - 5 } & $1.57 \pm 0.4 * * *$ & $18.9 \pm 2.3^{*}$ & $1.05 \pm 0.15^{* *}$ & $15.3 \pm 2.51^{*}$ \\
\hline \multirow{2}{*}{$\mathbf{3 2}$} & $1.3-2.4$ & $24.6-27.9$ & $1.1-1.3$ & $15.8-19.0$ \\
\cline { 2 - 5 } & $1.6 \pm 0.64 *$ & $25.7 \pm 1.63^{*}$ & $1.2 \pm 0.06^{* * *}$ & $17.9 \pm 1.5 * *$ \\
\hline \multirow{2}{*}{$\mathbf{3 6}$} & $1.5-1.86$ & $24.0-29.2$ & $1.3-1.8$ & $18.1-9.8$ \\
\cline { 2 - 5 } & $1.73 \pm 0.17^{* *}$ & $26.4 \pm 2.6^{* *}$ & $1.36 \pm 0.28^{*}$ & $18.6 \pm 0.54 * *$ \\
\hline
\end{tabular}

Abbreviations: SPS, superior petrous sinus; IPS, inferior petrous sinus ${ }^{\mathrm{a}}$ Mean $(\mu) \pm \mathrm{SD}(\sigma \eta-1), \mathrm{mm}$

Significance levels: ${ }^{* * *} \mathrm{p}<0.001 ;{ }^{* *} \mathrm{p}<0.01 ;{ }^{*} \mathrm{p}<0.5$; NS, no statistical significance

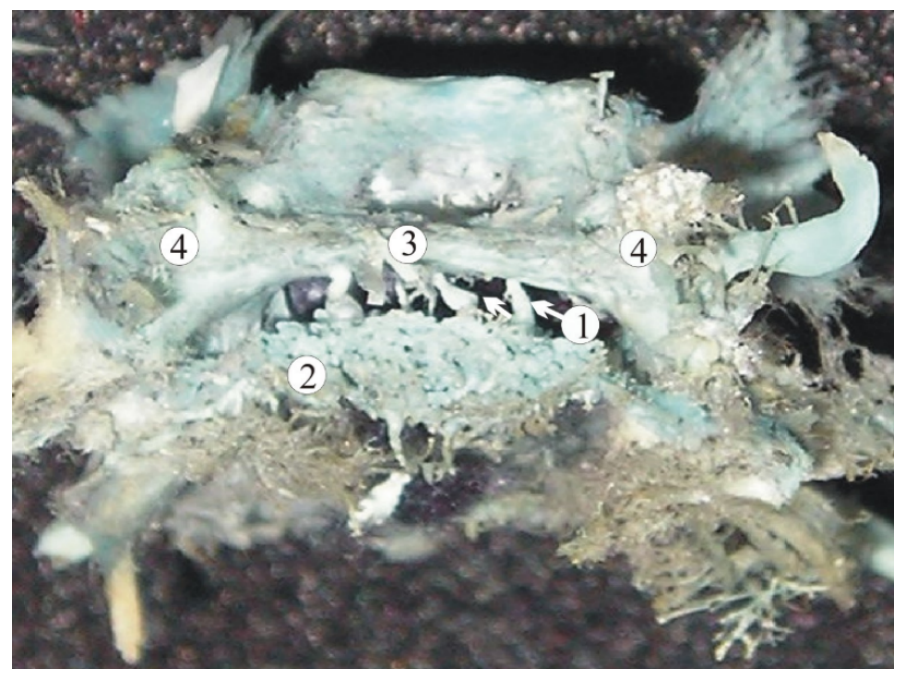

Figure 3. A plastic cast of the parasellar dural sinuses of a 20 -week human fetus showing the anterior cavernous sinus (3) communicating with the sphenoid venous plexus (2) via several emissary veins (1). Other abbreviations: 4 , cavernous sinus 


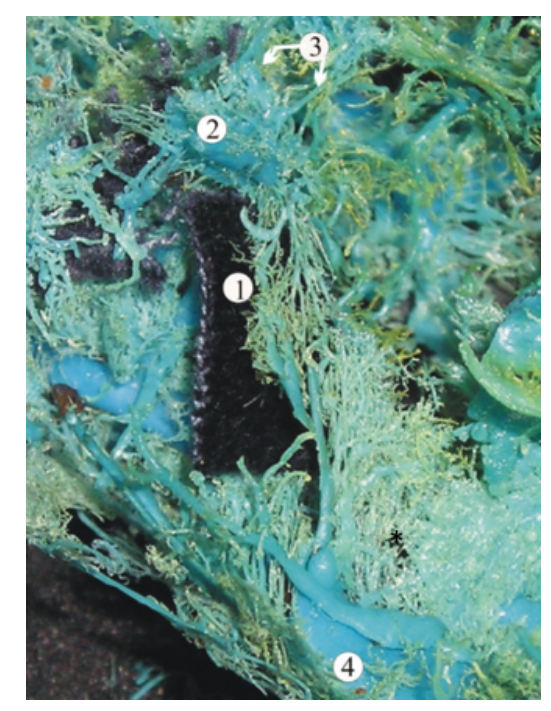

Figure 4. A plastic cast of the dural venous complex of the skull base of a 20 -week human fetus. The superior petrous sinus (1) has a branching pattern; the intra-dural venous plexus of the tentorium cerebellum $(*)$ is drained by the sinus via several dural veins. Other abbreviations: 2 , cavernous sinus; 3 , anterior and posterior intercavernous sinuses; 4 , sigmoid sinus

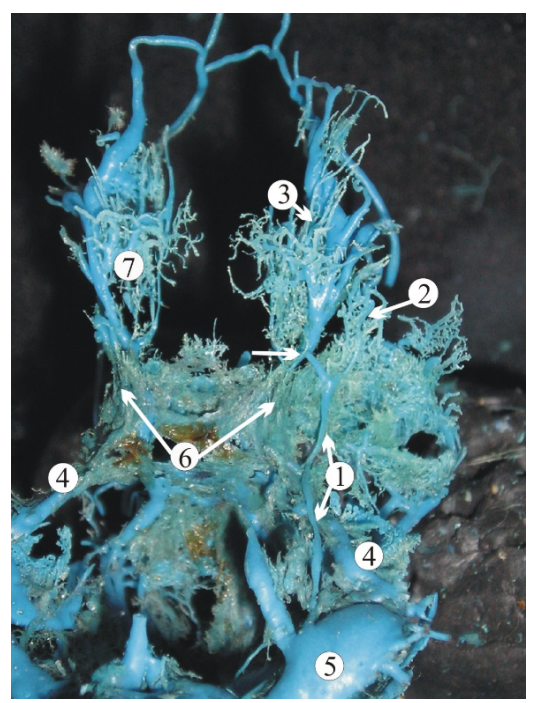

Figure 5. A plastic cast of the cavernous dural complex of a 24-week human fetus showing the relationship of the superficial middle cerebral vein (1) with the superior petrous sinus (4). The superior ophthalmic vein (3) contributes to the formation of the superficial middle cerebral vein (white arrow). Other abbreviations: 5 , sigmoid sinus; 6 , cavernous sinus; 7, orbital plexus

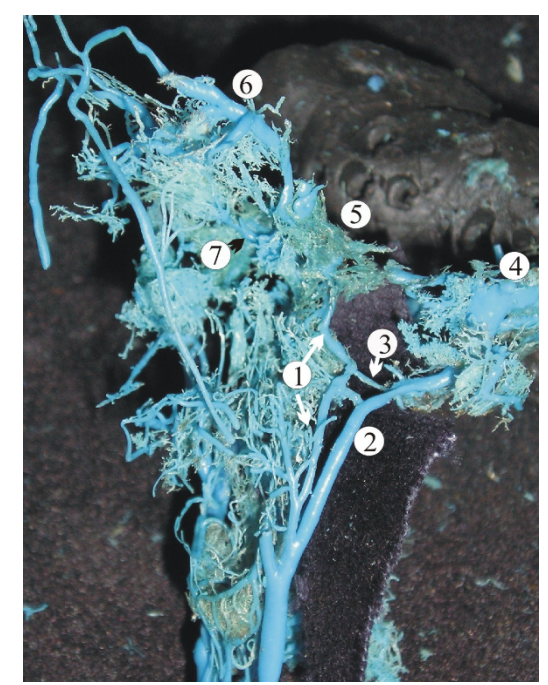

Figure 6. A plastic cast of the dural sinuses of the skull base of a 28 -week human fetus revealing the extracranial extension of the inferior petrous sinus (1) which parallels the internal jugular vein (2) and anastomoses with it via the emissary veins within the jugular foramen (3). The other abbreviations are: 4 , sigmoid sinus; 5 , cavernous sinus; 6 , superior ophthalmic vein; 7, pterygoid venous plexus 


\section{Discussion}

The plasticity of the fetal cranial venous system is an essential prerequisite for maintaining adequate blood circulation in cerebral hemispheres under conditions of their intensive development. The compensatory mechanisms of the cerebral venous outflow are designed to prevent complete obstruction caused by congenital or traumatic occlusion of one or several venous channels. Among two anatomic types of fetal dural sinus malformations, the lateral ones, affecting one of the jugular bulbs and adjacent sinuses, are considered to be benign in comparison with the midline malformations, involving the confluence of sinuses; this fact was explained by compensatory drainage of the brain via the dural sinuses of the opposite side of the skull [9]. In his research on dural arteriovenous shunts in neonates, M.P. Souza highlighted that neurological symptoms of this pathology largely depend on maturation and by-pass abilities of the cavernous sinus [4]. Barbosa, et al. analyzed 30 clinical cases of the dural arteriovenous shunts diagnosed predominantly during the first year of life; 8 out of 30 cases were diagnosed intrauterine employing ultrasound techniques. In his observation, 12 patients had an unfavorable neurological outcome and died, while the other 18 babies underwent endovascular treatment and survived with good neurological results [3]. Although the dural malformations and arteriovenous fistulas in neonates are barely studied and rarely reported, they may be timely diagnosed and successfully treated [5]. Thus, a deep understanding of the anatomical features of the fetal venous dural system and ability to analyze the processes of fetal maturation and establishment of compensatory potentials of the dural sinuses of the skull base are clinically relevant.

\section{Review of embryologic development}

The complex processes of formation and reconstruction of the primary venous plexuses are induced and influenced by a number of genetic factors involved in the regulation of the vascular plasticity during early embryogenesis. In the experiment on gene transcripts of mice and zebrafish, Herper RL has distinguished 61 genes with the expression pattern closely linked to the process of development of the circulatory system [10].

The cranial venous system begins to differentiate in $4 \mathrm{~mm}$ embryo and originally is presented by an anterior, middle and posterior cranial venous plexuses all drained via a single primary head vein (PHV) that contributes to the anterior cardinal vein. In the $14 \mathrm{~mm}$ embryo, the cranial venous plexuses show a well-distinguished three-layered structure. The middle layer develops into the dural venous system, while the superficial and deep layers contribute to the veins of the soft tissues and brain, respectively. In the next stages of development, the anastomotic loops between these primary layers will form emissary and communicative veins $[1,2]$.

The major reconstruction of the dural venous system happens in the $18 \mathrm{~mm}$ embryo, when the enlargement of the otic capsule and growth of the brain put mechanical force on surrounding tissues and induce a passive migration of developing dural channels. That leads to a separation of zones of importance along the PHV. The cranial part of the vein takes responsibility for the drainage of the orbits and developing eyes, while the anterior, middle and posterior dural plexuses start to drain into its caudal part. Subsequently, the anterior part of the PHV gives origin to the cavernous sinus, while the caudal part evolves into the internal jugular vein $[1,2]$. It should be noted that the main dural channels of the skull base have formed and acquired the final form already in the embryonic period. This complex is ahead in development than the sinuses of the calvaria and extracranial venous plexuses, which indicates its phylogenetic antiquity.

\section{Early fetal period of development (16-24 weeks)}

Present research reveals that the dural venous complex of the skull base approximates typical structure at the beginning of the early fetal period. The anterior and posterior intercavernous sinuses connect the lateral compartments of the cavernous sinus, closing the venous circle around the sella turcica.

At 16 weeks of gestation, an overall diameter of the lateral compartments of the cavernous sinus reaches $3.2 \mathrm{~mm}$, approximately equal to the sum of the widths of the upper and lower petrosal sinuses (Tables 1 and 2). Despite a relatively large diameter, the cavernous sinus does not take significant intracranial tributaries at that time. However, orbital trunks $(0.4-0.6 \mathrm{~mm})$ formed by the confluence of veins of the orbit enter the anterior part of the cavernous sinus (Figure 7). Therefore, the primary function of the dural venous complex of the skull base at this point is to drain well-developed venous plexus of orbit towards the internal jugular vein.

In fetuses 20-22 weeks of gestation, a significant increase of the cerebral venous outflow from the intensively growing cerebral hemispheres limits drainage capacity of the basilar venous complex, complicating the local hemodynamics. That induces the development of intracranial tributaries of the cavernous sinus, including the superficial middle cerebral vein, and provokes the formation of accessory dural sinuses of the skull base such as spheno-temporal, orbito-petrosal, and orbito-transversal sinuses, which rarely persist in adults (Figure 8). Simultaneous formation of the venous plexuses of the foramen ovale, rotundum, and spinosum provides collateral venous outflow from the cranial cavity, connecting the cavernous sinus with the extra-cranial venous plexuses, particularly, with the pterygoid venous plexus (Figure 2). Some efferent vessels from the pterygoid plexus enter the transverse facial and retromandibular veins contributing to the external jugular vein, which is sufficiently formed by this stage.

The volume of venous outflow from the intracranial structures reaches peak levels at 24 weeks of gestation. Morphologically, it is manifested by establishment of secure connections between the dural venous sinuses of the skull base and the extracranial venous vessels. Together they form a unified basal venous complex, comprising the venous plexuses of the orbits, the emissaries of the skull base, and such venous plexuses as the pterygoid, retropharyngeal, and paravertebral (Figure 9). This complex represents an anatomical foundation of the basilar path of collateral venous circulation, which joins main tracks of venous outflow from the head and brain, namely the systems of the external and internal jugular veins with the vertebral veins.

\section{Late fetal period of development (28-36 weeks)}

The enlargement of the lumens of the cavernous and petrous dural sinuses notably slows down at 28-36 weeks of gestation due to relative stabilization of the pace of brain growth. As a result, we observe the particular reduction of the canals in the basilar venous complex, merging of the tiny vessels into the larger ones, and lessening of the density of the cerebral venous tributaries of the cavernous sinus.

The gradual reduction of the primary venous plexuses continues through the whole third trimester at the genetically determined individual pace, laying down a wide range of anatomic variability. Two marginal types of the variability of the dural venous complex of the skull base became evident by the end of the antenatal period. The first type featured a considerable reduction of the primary venous system 
leading to the formation of one-canal shaped dural venous sinuses, lower number of tributaries, and diminishing density of the extracranial venous plexuses; it was registered in $21.3 \%$ of 36 -week fetuses. In this group, the average diameter of the cavernous sinus was equal to 1.45 $\pm 0.63 \mathrm{~mm}$, while widths of the superior and inferior petrous sinuses constituted $1.16 \pm 0.11$ and $1.14 \pm 0.05 \mathrm{~mm}$, respectively. The emissary veins of the foraminae ovale, rotundum, and spinosum were well determined and had a diameter from 0.4 up to $1.6 \mathrm{~mm}$. The second marginal type of variability was observed in $32 \%$ of fetuses. It featured the enhanced venous basal complex typical for the early fetal period, with the multi-canal shape of dural sinuses, and an abundance of tributaries and anastomoses. In this study group, the average width of the cavernous sinus reached $2.48 \pm 0.8 \mathrm{~mm}$, and the diameter of the superior petrous sinus was up to $1.78 \pm 0.94 \mathrm{~mm}$, while the inferior

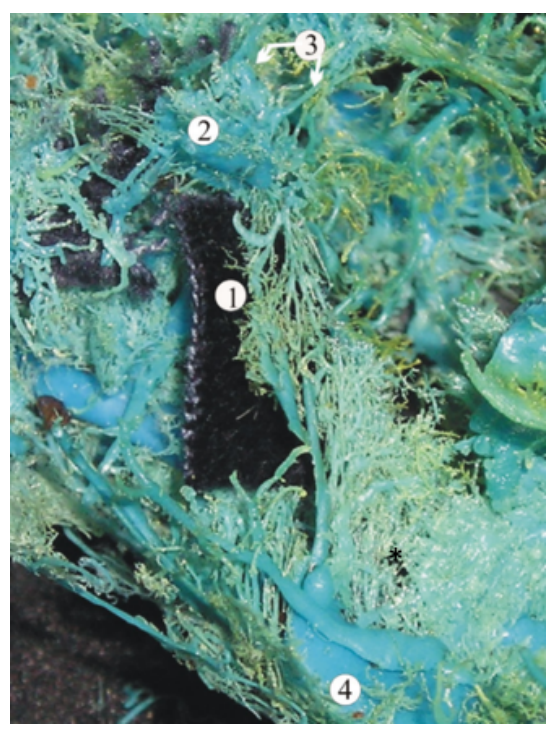

Figure 7. A plastic cast of the dural venous complex of the skull base of a 16-week fetus showing pronounced development of the orbital venous plexus. Inferior ophthalmic vein (4) anastomoses with the deep veins of the face and with the superior ophthalmic vein (5) forming the common ophthalmic vein (3), which enters the cavernous sinus (2). The other abbreviations are: 3 , intercavernous sinuses; 6 , superior petrous sinus

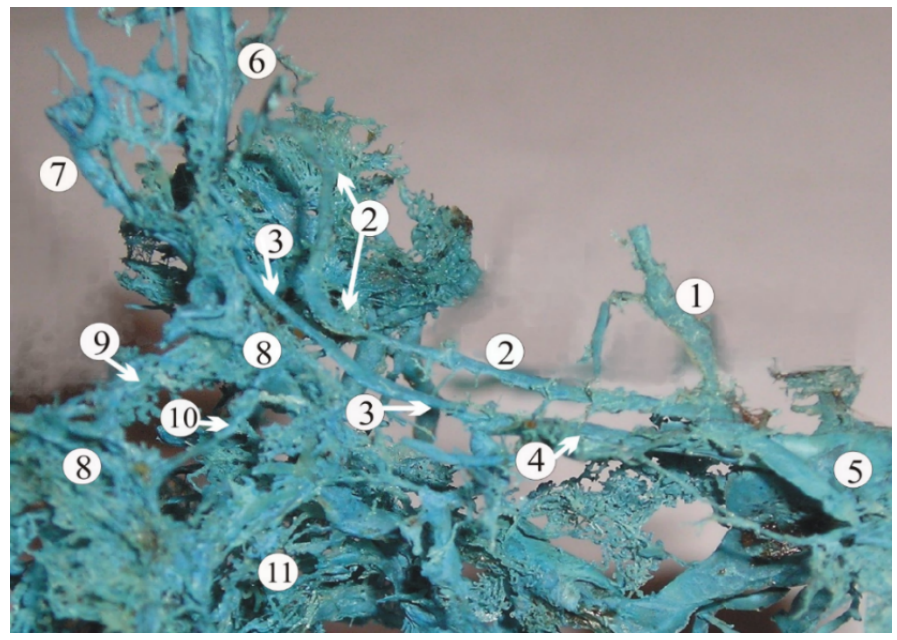

Figure 8. A plastic cast of the dural venous complex of a 24 -week fetus, showing the formation of the accessory venous routs of the skull base such as petro-squamous sinus (1), superficial middle cerebral vein (2), and orbito-petrous sinus (3). The other structures marked of this picture are: 4, superior petrous sinus; 5 , transverse sinus; 6 , superior ophthalmic vein; 7 , inferior ophthalmic vein; 8 , cavernous sinus; 9 , anterior intercavernous sinus; 10 , posterior intercavernous sinus; 11 , basilar venous plexus

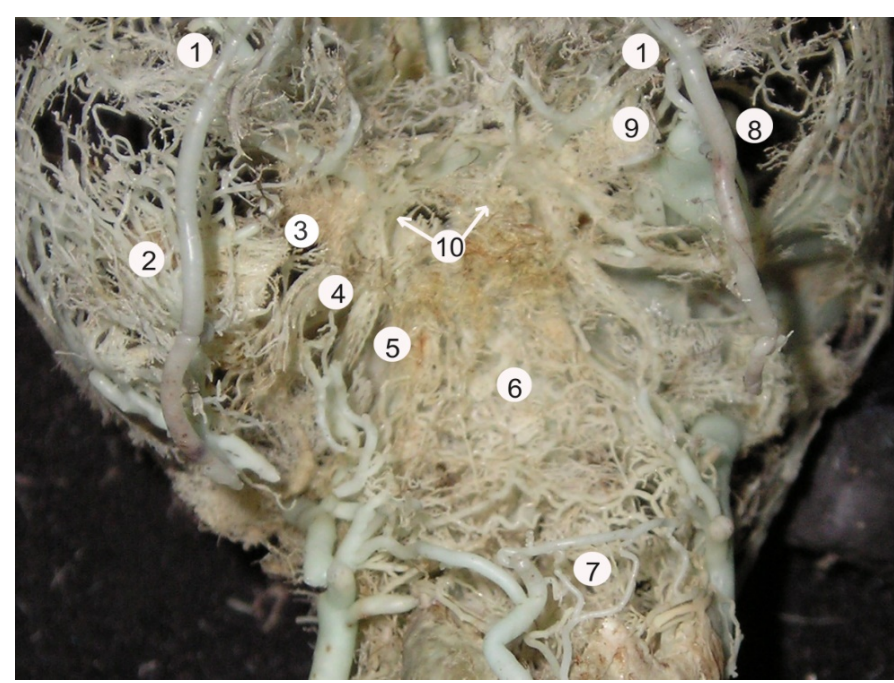

Figure 9. A plastic cast of the basal venous path of collateral circulation of a 28 -week fetus, which includes the orbital plexus (1), pterygoid venous plexus (2), emissary of skull base (3), carotid venous plexus (4), basilar venous plexus (5), retropharyngeal venous plexus (6), paravertebral venous plexus (7), anastomoses of the inferior ophthalmic vein $(8,9)$, and cavernous sinus (10)
Type I
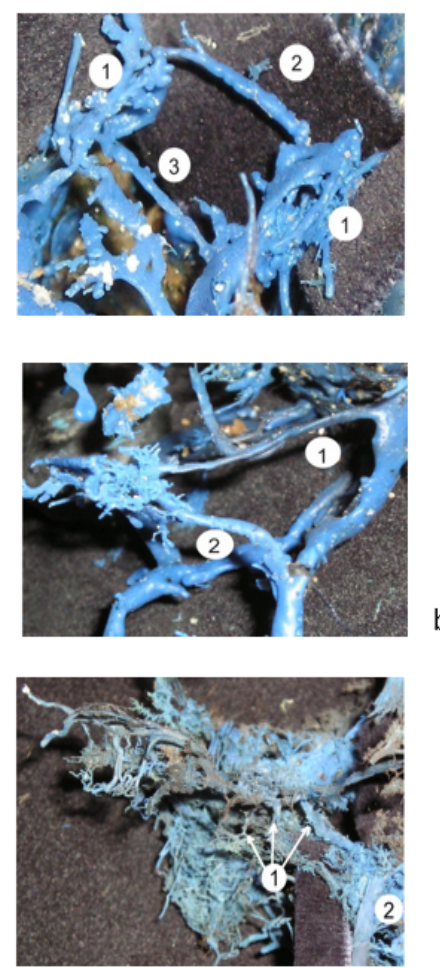

Type II
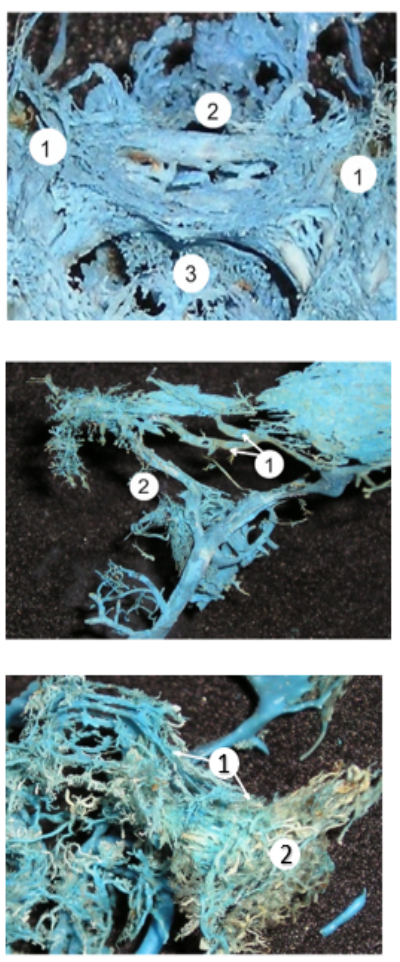

Figure 10. The morphological comparison of two marginal types of variability of the dural venous system of the skull base in 36-week human fetuses; a) the parasellar venous complex consisting of cavernous sinuses (1), anterior (2), and posterior (3) intercavernous sinuses; b) the superior (1) and inferior (2) petrous sinuses; c) emissaries of foraminae rotundum, ovale, and spinosum (1) connecting the cavernous sinus with the pterygoid venous plexus (2). The morphological characteristics of each type are presented in Table 3

petrous sinus was $1.38 \pm 0.27 \mathrm{~mm}$ in diameter (Table 3 ). The emissaries of the skull base were formed by numerous vessels less than $0.1 \mathrm{~mm}$ in diameter, which widely communicated with extracranial venous plexuses providing a robust bypass for the cerebral venous outflow (Figure 10). The intermediate forms filled the interval between the 
Table 3. Characteristics of the marginal types of variability registered in 36-week human fetuses

\begin{tabular}{|c|c|c|c|c|c|c|c|c|}
\hline \multirow{3}{*}{$\begin{array}{r}\begin{array}{r}\text { Type of } \\
\text { variability }\end{array} \\
\text { mean } \pm \mathrm{SD},\end{array}$} & \multirow{3}{*}{ Type I } & \multirow{3}{*}{ Type II } & \multicolumn{6}{|c|}{ Paired-samples t-test } \\
\hline & & & \multicolumn{2}{|c|}{$\begin{array}{l}\text { 95\% Confidence interval of the } \\
\text { difference }\end{array}$} & \multirow{2}{*}{ Std. Error mean } & \multirow[t]{2}{*}{$t$} & \multirow{2}{*}{$d f$} & \multirow{2}{*}{ Sig. (2-tailed) } \\
\hline & & & Lower & Upper & & & & \\
\hline $\mathrm{CS}, d$ & $1.45 \pm 0.63$ & $2.48 \pm 0.8$ & -1.22714 & -0.89247 & 0.08335 & -2.795 & 24 & 0.013 \\
\hline $\mathrm{CS}, l$ & $7.55 \pm 0.92$ & $6.28 \pm 1.49$ & 1.27143 & 1.53687 & 0.06614 & 3.423 & 24 & 0.032 \\
\hline SPS, $d$ & $1.16 \pm 0.11$ & $1.78 \pm 0.94$ & -0.60385 & -0.47743 & 0.03183 & -3.785 & 24 & 0.001 \\
\hline SPS, $l$ & $30.3 \pm 5.49$ & $26.24 \pm 3.8$ & 3.51096 & 4.46664 & 0.23152 & 1.722 & 24 & 0.104 \\
\hline IPS, $d$ & $1.14 \pm 0.05$ & $1.38 \pm 0.27$ & -0.26789 & -0.16251 & 0.02553 & -2.437 & 24 & 0.023 \\
\hline IPS, $l$ & $19.8 \pm 0.83$ & $17.9 \pm 1.3$ & 1.89064 & 2.14936 & 0.06268 & 3.829 & 24 & 0.001 \\
\hline
\end{tabular}

CS, cavernous sinus; SPS, superior petrous sinus; IPS, inferior petrous sinus; d, diameter; 1, length; $t$, $t$ value; df, degree of freedom ( $n=25)$; Sig, significance level. The mean difference is statistically significant at $\mathrm{p}<0.05$

marginal types establishing the wide range of variability of the dural venous complex of the skull base.

Thus, the remodeling of the dural venous complex of the skull base continues through the whole antenatal period of human development. The type of variability and compensatory potential of the drainage system of the brain primarily depends on the genetically determined intensity of the reduction of the venous plexuses and dural sinuses of the skull base during the late fetal period of human development.

\section{References}

1. Streeter GL (1915) The development of the venous sinuses of the dura mater in the human embryo. Am J Anat 18: 145-178.

2. Sadler TW (2011) Langman's Medical Embryology. (13th Edn), Lippincott William and Wilkins.

3. Barbosa M, Mahadevan J, Weon YC, Yoshida Y, Ozanne A, et al. (2003) Dural Sinus Malformations with Giant Lakes in Neonates and Infants. Interv Neuroradiol 9: 407-424.
4. Souza S, Willinsky RA, Terbrugge KG (2003) Intracranial Dural Arteriovenous Shunts in Children. Interv Neuroradiol 9: 47-52.

5. Lasjaunias PL, TerBrugge K (1997) Vascular Diseases in Neonates. Infants and Children. Interventional Neuroradiology Management, ed. PL Lasjaunias. Paris: Springer-Verlag Berlin Heidelberg.

6. Kiyosue H, Mori H, Sagara Y, Hori Y, Okahara M, et al. (2009) Basal cerebral venous drainage from cavernous sinus dural arteriovenous fistulas. Neuroradiology 51: 175181.

7. Theaudin M, Saint-Maurice JP, Chapot R, Vahedi K, Mazighi M, et al. (2007) Diagnosis and treatment of dural carotid-cavernous fistulas: a consecutive series of 27 patients. J Neurol Neurosurg Psychiatry 78: 174-179.

8. Suzuki Y, Matsumoto K (2000) Variations of the superficial middle cerebral vein: classification using three-dimensional CT angiography. Am J Neuroradiol 21: 932-938.

9. McInnes M, Fong K, Grin A, Brugge K, Blaser S, et al. (2009) Malformations of the Fetal Dural Sinuses. Can J Neurol Sci 36: 72-77.

10. Herper RL (2010) Genetic regulation of vascular development. Royal Netherlands Academy of Art and Sciences (KNAW), Rotterdam, Netherlands.

Copyright: @2019 Kornieieva M. This is an open-access article distributed under the terms of the Creative Commons Attribution License, which permits unrestricted use, distribution, and reproduction in any medium, provided the original author and source are credited. 